\title{
Жаталов В.В. ", аспирант,
}

Белгородский государственный технологический университет им. В.Г. Шухова, Россия

*Ответственный автор E-mail: shatalov-victor@bk.ru

\section{АКТУАЛЬНОСТЬ СОЗДАНИЯ ИСКУСТВЕННЫХ ДЫХАТЕЛЬНЫХ АТМОСФЕР В УСЛОВИЯХ ЗАГРЯЗНЕНИЯ АТМОСФЕРНОГО ВОЗДУХА}

\begin{abstract}
Аннотация: в настоящее время остро встала проблема качества воздуха в крупных городах с большим количеством автомобилей и промышленных предприятий. Нормативные документы на основе которых проектируют системы вентиляции регламентирует очистку наружного воздуха от пыли. Очистка от газообразных загрязнений, а так же от взвешенных аэрозолей данные документы не регламентируют. По результатам исследования качества воздуха в Москве за последние несколько лет зарегистрировано повышенное содержание загрязняющих веществ, таких как $\mathrm{NO}, \mathrm{CO}, \mathrm{CO}_{2}, \mathrm{SO}_{2}$. Вблизи оживленных магистралей в «часы пик» автомобили локально «выжигают» кислород воздуха, а выделяемые продукты горения ещё больше снижают удельную концентрацию кислорода в воздухе. Так же, большое количество загрязняющих веществ поступает от промышленных и сельскохозяйственных предприятий. Применение в системах вентиляции дополнительных фильтров очистки, таких как фотокаталитический фильтр, адсорбционный фильтр способно снизить концентрации газообразных загрязнений до приемлемого минимума, но проблему пониженного содержания кислорода они не решат. Вдыхание воздуха с превышением ПДК в течении длительного времени может привести к необратимым последствиям для здоровья. В связи с этим, становится актуальной задача создания искусственных дыхательных атмосфер. Наибольшую актуальность качество воздуха и достаточное содержание кислорода имеет для детских учреждений, а так же специальных служб, работа которых связана с повышенной концентрацией внимания. Проблема загрязнения воздуха аэрозолями и мелкодисперсной пылью стала особо актуальной в связи со значительным увеличением заболеваний дыхательного тракта и различными аллергиями.
\end{abstract}

Ключевые слова: загрязнение воздуха, искусственные дыхательные атмосферы

\section{Введение}

Проектирование систем вентиляции осуществляется в соответствии с СП 60.13330.2012 «Отопление, вентиляция и кондиционирование воздуха». Исходя из пункта 7.1.1 «Вентиляцию следует применять для обеспечения параметров микроклимата и качества воздуха в пределах допустимых норм». А так же в соответствии с пунктом 7.1.17. «Очистка воздуха от пыли в системах механической вентиляции и кондиционирования должна обеспечивать содержание пыли в подаваемом воздухе не более: а) ПДК в атмосферном воздухе населенных пунктов - при подаче его в помещения жилых и общественных зданий; б) $30 \%$ ПДК в воздухе рабочей зоны - при подаче его в помещения производственных и административно-бытовых зданий; в) 30\% ПДК в воздухе рабочей зоны для частиц пыли размером не более 10 мкм - при подаче его в кабины крановщиков, пульты управления, зону дыхания работающих, а также при воздушном душировании; г) допустимых концентраций по техническим условиям на вентиляционное оборудование и воздуховоды. [1].

СП 60.13330.2012 регламентирует очистку наружного воздуха от пыли, но не от газообразных загрязнений. Как показывают исследования атмосферного воздуха за последние несколько лет в г. Москва, превышения загрязнений в газообразной форме, такие как $\mathrm{NO}, \mathrm{CO}, \mathrm{CO}_{2}, \mathrm{SO}_{2}$ близок к ПДК либо превышает его.

\section{Методы и материалы}

Повышенное внимание к аэрозольному загрязнению атмосферного воздуха появилось после выявления взаимосвязи некоторых болезней дыхательной системы с повышенным содержанием взвешенных частиц в атмосферном воздухе, а также повышение негативного эффекта при превышении концентрации газообразных загрязнителей [6]. По результатам медицинских исследований ВО3 внесла взвешенные частицы размером менее 10 мкм (РМ10) в перечень загрязняющих веществ атмосферного воздуха концентрацию которые нужно контролировать [5].

При разработке систем вентиляции жилых и административных помещений необходимо применять не только системы фильтрации от пыли, но и системы очистки от газообразных загрязнений не учтенных в СП. Так же необходимо учитывать синергетический эффект при присутствии нескольких загрязнителей. 


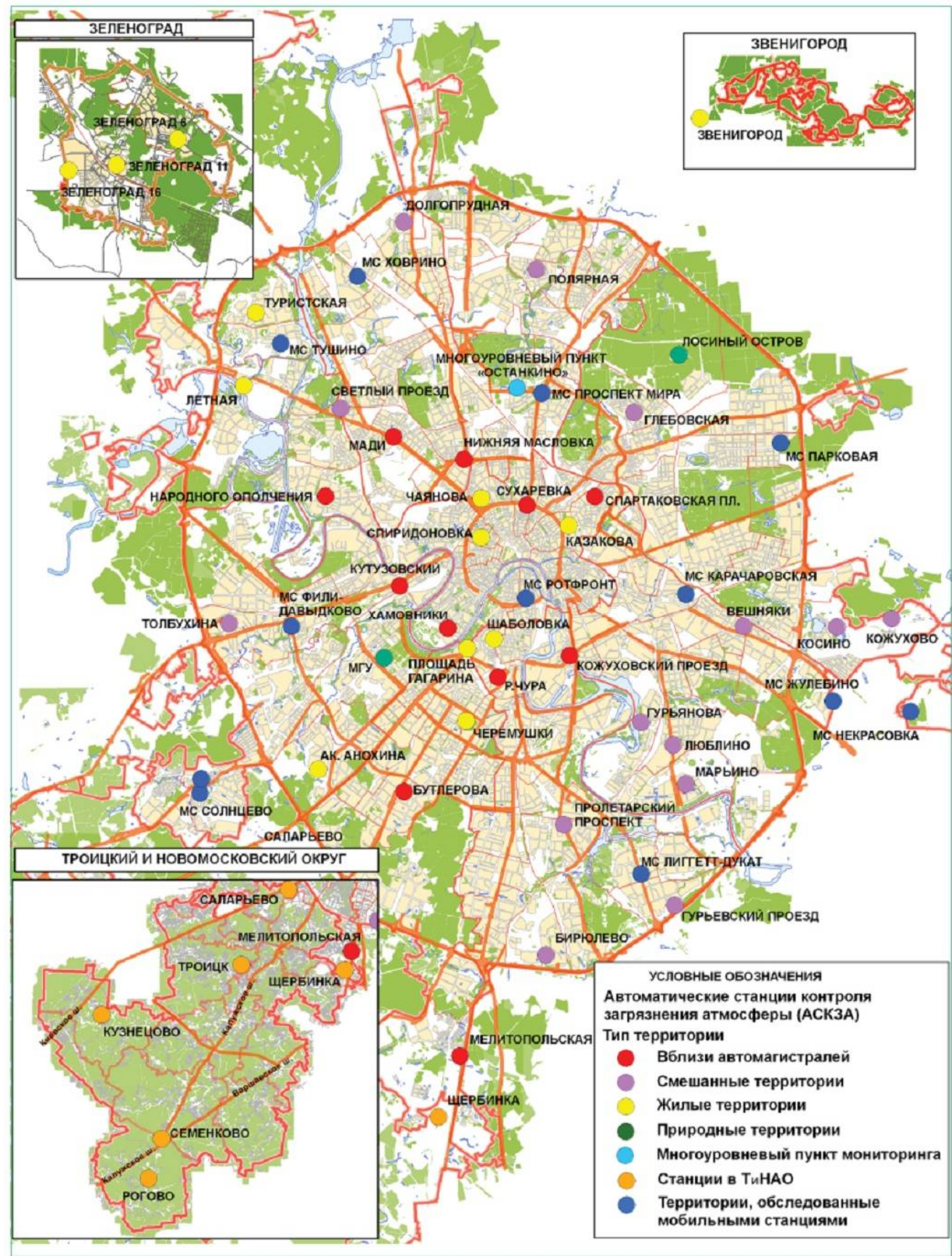

Рис. 1. Схема расположения автоматических станций контроля загрязнения атмосферы в Москве и пригородах

Fig. 1. Arrangement of automatic monitoring stations of air pollution in Moscow and its suburbs

По данным Госкомстата России порядка 50 млн. человек в РФ проживают в условиях 10-кратного превышения вредных веществ в атмосфере, порядка 60 млн. проживают в условиях 5-кратного превышения концентрации. По статистике Госкомстата России в период с 1990 по 1999 среднее значение выбросов загрязняющих веществ в атмосферу значительно снизился ввиду спада промышленного производства. За тот же период концентрации $\mathrm{CO}, \mathrm{CO}_{2}, \mathrm{NO}$ выросло на $15 \%$ ввиду роста количества автомобилей. В целом даже после снижения вредных выбросов от промышленных предприятий уровень загрязнения многих городов остается недопустимо высоким [7].

Автомобильный транспорт является основным источником загрязняющих веществ как для Московского региона в частности, так и для России в целом. На рис. 2 статистика роста количества автомобилей в Москве за 2000-2015 г. 


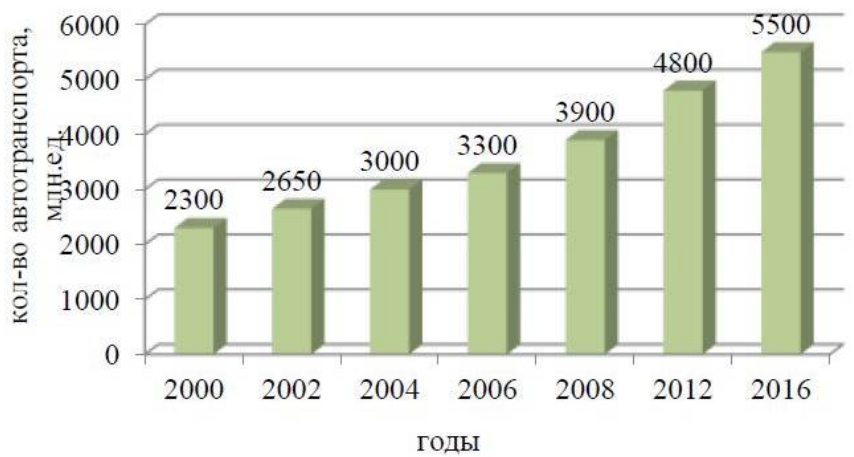

Рис. 2. Рост количества автомобилей в г. Москва за период 2000-2015 г.

Fig. 2. Increase in the number of cars in Moscow for the period 2000-2015

Увеличение количества автомобильного транспорта, большой количество перекрестков приводит к снижению средней скорости движения, образованию «пробок». Данные обстоятельства усугубляют экологическую обстановку т.к. максимальное количество вредных выбросов происходит при небольших скоростях движения, а так же на «холостом ходу».

Ухудшение экологической обстановки крайне отрицательно сказывается на заболеваемости органов дыхания у взрослых и детей! За период с 2005 по 2014 годы количество заболевших хроническим бронхитом взрослого населения возросло в 1,5 раза. [8].

Диоксид азота обладает аллергическим действием на организм человека, особенно чувствительны к его воздействию дети. Статистика показывает рост заболеваемости бронхиальной астмой, детей с ростом концентрации диоксида азота в воздухе. Отмечается так же рост на 40-60\% заболеваний острым бронхитом у детей [9].

Основные газообразные вещества загрязняющие воздух, которые необходимо учитывать при разработке систем вентиляции:, $\mathrm{CO}, \mathrm{CO}_{2}, \mathrm{SO} 2, \mathrm{NO}$, а так же летучие органические соединения (бензельной группы, растворители и пр.).

Качество воздуха в помещении, которое обеспечивает приточная вентиляция должно соответствовать нормам СП, так же необходимо учитывать загрязнения от людей, технологического оборудования, мебели в помещении и пр.

При составлении Т3 на разработку систем приточной вентиляции необходимо учитывать не только загрязнения приточного воздуха но и загрязнения выделяемые в самом помещении [2].

\section{Результаты и обсуждения}

Основные газообразные вещества загрязняющие атмосферный воздуха в г. Москва сведены в табл. 1 . Данные собраны автоматическими станциями контроля загрязнения атмосферного воздуха (АСКЗА) расположенными в разных точках г. Москвы и пригородах (рис. 1) [3]

Таблица 1

\section{Среднегодовые концентрации основных загрязняющих веществ в 2014 г превышающие ПДК}

Average annual concentrations of main pollutants in 2014 exceeding MPC

Table 1

\begin{tabular}{|c|c|c|c|}
\hline $\begin{array}{c}\text { Загрязняющее } \\
\text { вещество }\end{array}$ & $\begin{array}{c}\text { Среднее значение } \\
\text { вредных веществ } \\
\text { в долях ПДК сс }\end{array}$ & $\begin{array}{c}\text { Диапазон концентраций } \\
\text { вредных веществ } \\
\text { в долях ПДК сс }\end{array}$ & $\begin{array}{c}\text { Наибольшая повторяемость } \\
\text { превышения максимальной } \\
\text { разовой ПДК, \% }\end{array}$ \\
\hline $\mathrm{CO}$ & 0,15 & $0,06-0,44$ & 0,48 \\
\hline $\mathrm{NO}_{2}$ & 0,95 & $0,51-2,12$ & $\mathbf{0 , 2 9}$ \\
\hline $\mathrm{NO}$ & 0,43 & $0,19-1,55$ & 0,93 \\
\hline $\mathrm{H}_{2} \mathrm{~S}$ & - & - & 4,52 \\
\hline $\mathrm{O}_{3}$ & 0,96 & $0,66-1,25$ & 0,31 \\
\hline $\mathrm{C}_{6} \mathrm{H}_{5} \mathrm{OH}$ (фенол) & 0,4 & $0,33-1,00$ & $\mathbf{0}$ \\
\hline Стирол & 1,74 & $0,7-2,7$ & $\mathbf{0 , 1 0}$ \\
\hline
\end{tabular}

В случае если в помещении содержатся несколько загрязняющих веществ однонаправленного воздействия, то при суммировании отношений фактических концентраций каждого из них (К1, К2,..., Кn) в воздухе рабочей зоны к их ПДК (ПДК1, ПДК2 ... ПДКп) должна быть менее 1 (1) 


$$
\frac{K_{1}}{\Pi Д K_{1}}+\frac{K_{2}}{\Pi Д \kappa_{2}}+\ldots+\frac{K_{n}}{\Pi Д \kappa_{n}} \leq 1
$$

Таким образом, если в воздухе подаваемом в помещение просуммировать отдельные ПДК загрязняющих веществ, суммарный ПДК превысит 1, из этого можно сделать вывод что приточный воздух подаваемый в помещения без специальной очистки уже превышает ПДК, а смешавшись с загрязнениями в самом помещении значительно превысит ПДК.

Из вышеизложенного можно сделать вывод, что воздух в таких помещениях (вблизи загруженных автомагистралей г. Москвы) может оказывать прямое или косвенное вредное воздействие на организм человека в условиях неопределённо долгого круглосуточного вдыхания.

Решение данной проблемы были найдены в изобретениях описываемых в патентах №2 431784 и №2 484 384 рис. 3, 4. Суть данных изобретений сводится к разложению входящего воздуха на составляющие компоненты (кислород и азот) на поверхностях селективных мембран. И дальнейшее смешивание разделенных компонентов в нужной пропорции для получения дыхательной атмосферы благоприятной для жизнедеятельности человека без вредных примесей из наружного воздуха.

Значительным недостатком этих решений является то, что практически весь объем воздуха необходимого для вентиляции помещения через компрессор, предварительно очистив высокоэффективными фильтрами. Перед подачей воздуха на селективные мембраны его необходимо предварительно осушить для эффективной фильтрации компонентов. Для реализаций этих решений необходимо оборудование имеющее очень высокую стоимость, значительные габариты и массу. К тому же при работе компрессор создает высокий уровень шума и потребляет значительное количество электрической энергии. Из всего вышеизложенного можно сделать вывод: практическая реализация данного проекта экономически невыгодна из за крайне высоких капитальных вложений, а так же высоких текущих затрат на электроэнергию.

Приемлемым решением данной задачи, является использование очищенного рециркулируемого воздуха рис. 5. В классических системах вентиляции воздух удаляется из помещения по причине пониженного содержания кислорода после дыхания и повышенного содержания вредных примесей выделяемых мебелью, элементами отделки, приборами и оборудованием используемым людьми в жилых, офисных и производственных помещениях. Самый простой и дешевый способ создания благоприятной для человека атмосферы в помещениях является устройство приточно-вытяжной вентиляции, но это при условии, что наружный воздух фильтруемый и подаваемый в помещения содержит достаточное количество кислорода и не содержит вредных и токсичных примесей. Загрязненный наружный воздух очищать дорого, а при пониженном содержании кислорода, вообще не имеет смысла подавать в помещения.

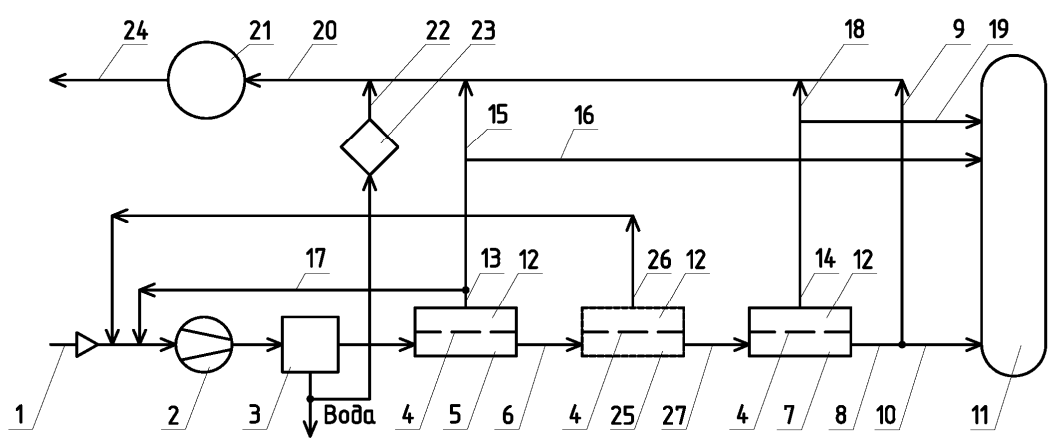

Рис. 3. Патент №2431784, Кл. F24F 3/14

Fig. 3. Patent No.2431784, Cl. F24F 3/14

1 наружный воздух, 2 компрессор, 3 осушитель, 4 поверхность селективной мембраны, 5 дополнительный мембранный модуль, 6 газовый поток в области высокого давления после дополнительного модуля, 7 основной мембранный модуль, 8-10 газовый поток после основного мембранного модуля, 11 наружный воздух, 12 область низкого давления, 13 газовый поток после дополнительного мембранного модуля, 14 газовый поток после основного мембранного модуля, 15 часть газового поток после дополнительного мембранного модуля подаваемая в рабочее пространство, 16 часть газового поток после дополнительного мембранного модуля сбрасываемая в атмосферу, 17 часть газового поток после дополнительного мембранного модуля подаваемая во внешний газовый поток, 18 часть газового поток после основного мембранного модуля подаваемая рабочее пространство, 19 часть газового поток после основного мембранного модуля сбрасываемая в атмосферу, 20 питающий газовый поток, 21 рабочее пространство, 22 пары воды, 23 испа- 
ритель, 24 воздушный поток отводимый из рабочего пространства, 25 второй дополнительный мембранный модуль, 26 газовый поток после 2 дополнительного мембранного модуля, 27 газовый поток в области высокого давления после второго дополнительного модуля.

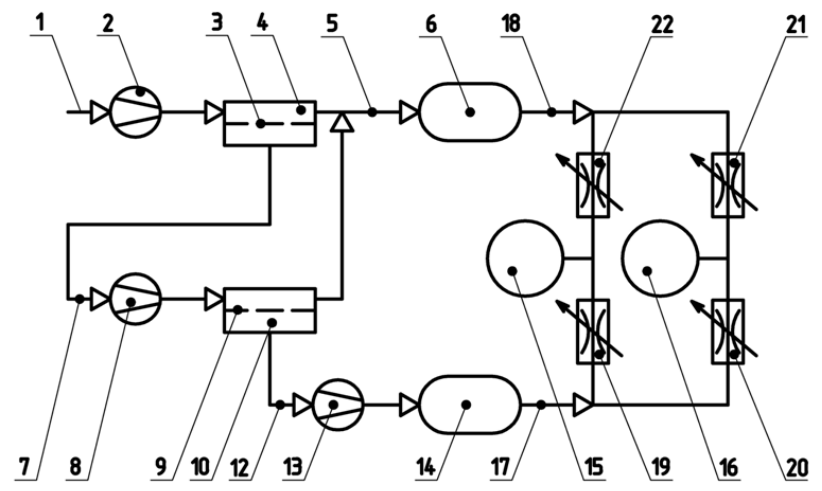

Рис. 4. Патент №2 484 384, Кл. F24F 3/14

Fig. 4. Patent №2 484 384, Cl. F24F 3/14

1 внешний газовый поток, 2 компрессор, 3, 9 поверхность селективных мембран, 4 мембранный модуль первой ступени, 5 газовый поток из области высокого давления, 6 промежуточная емкость, 7 газовый поток из области низкого давления мембранного модуля первой ступени установки, 8 компрессор, 10 мембранный модуль второй ступени, 11 газовый поток из области высокого давления мембранного модуля второй ступени установки, 12 газовый поток из области низкого давления мембранного модуля второй ступени установки, 13 компрессор, 14 промежуточная емкость, 15, 16 рабочие пространства, 17 газовый поток заданной величины с высоким содержанием кислорода, 18 газовый поток заданной величины с высоким содержанием азота, 19, 20, 21, 22 дроссели автоматической системы управления.

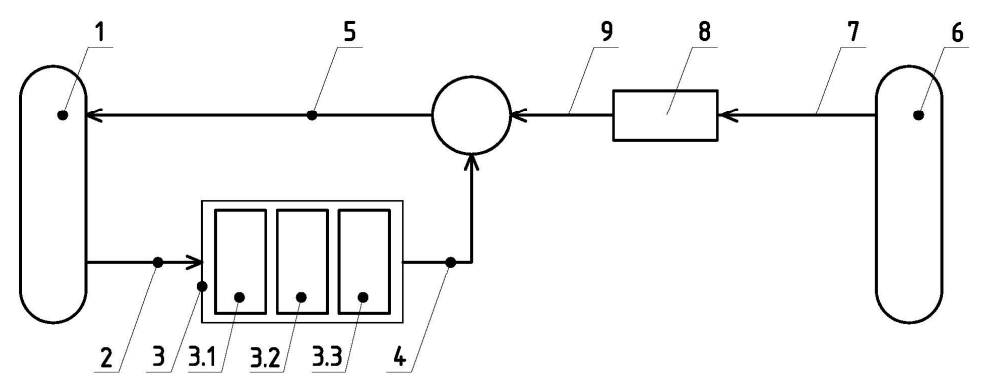

Рис. 5. Схема создания искусственной дыхательной атмосферы с молекулярной фильтрацией рециркулируемого воздуха

Fig. 5. Scheme of artificial respiratory atmosphere creation with molecular filtration of recirculated air

1 рабочая зона, 2 рециркулируемый воздух поступающий на очистку, 3 фильтрующая установка, 3.1 пылевой фильтр EU4, 3.2 фотокаталитический фильтр, 3.2 угольный фильтр, 4 очищенный рециркулируемый воздух, 5 очищенный рециркулируемый воздух обогащенный кислородом, 6 наружный воздух, 7 наружный воздух подаваемый для молекулярной фильтрации, 8 молекулярный фильтр, повышающий концентрацию кислорода до $\sim 90 \%, 9$ воздух, обогащенный кислородом до $\sim 90 \%$.

\section{Выводы}

Наиболее эффективным способом создания благоприятной дыхательной атмосферы в помещении при неблагоприятном составе наружного воздуха является система описанная на рис. 5. В выдыхаемом человеком воздухе содержится около 16\% кислорода. Поэтому есть смысл генерировать только 5\% от общего оборота воздуха в помещении, а остальные 95\% фильтровать установками, которые будут задерживать пыль, химические загрязнения а также разрушать бактерии и вирусы. При этом на генерацию 5\% кислорода понадобится на порядок менее производительное оборудование, а соответственно и на прядок менее дорогое по капитальным и текущим затратам, что делает данный проект экономически более эффективным.

\section{Благодарность}

Работа выполнена по гранту РНФ, проект №18-79-10025 


\section{Литература}

1. Свод правил СП 60.13330.2012 «Отопление, вентиляция и кондиционирование воздуха».

2. ГОСТ Р ЕН 13779-2007. «Вентиляция в нежилых зданиях. Технические требования к системам вентиляции и кондиционирования». С. $7-8$.

3. Доклад о состоянии окружающей среды в городе Москве в 2014 году / Под ред. А.О. Кульбачевского. М.: ДПиООС; НИА-Природа, 2015.С. $42-44$.

4. Межгосударственный стандарт ГОСТ 12.1.005-88 «Система стандартов безопасности труда (ССБТ). Общие санитарно-гигиенические требования к воздуху рабочей зоны (с Изменением N 1)». 7 с.

5. WHO 2005. Air Quality Guidelines: Global Update 2005. Particulate matter, ozone, nitrogen dioxide and sulfur dioxide // WHO. 2006. P. 484.

6. Воздействие взвешенных частиц на здоровье. Рекомендации в отношении политики для стран Восточной Европы, Кавказа и Центральной Азии // Всемирная организация здравоохранения. Европейское региональное бюро. 2013. С. 14. (www.euro.who.int).

7. Ушаков С.А., Кац Я.Г. Экологическое состояние территории России. М.: Academia, 2002. 127 с.

8. Владимиров С.Н. Транспортные заторы в условиях мегаполиса // Известия Московского государственного технического университета МАМИ. 2014. Т. 3. №1 (19). С. 77 - 84.

9. Протасов В.Ф. Экология, здоровье и охрана окружающей среды в России: учебное и справочное пособие. Финансы и статистика, 2009. 521 с.

\section{References}

1. Russian Building Code SP 60.13330.2012 «Otoplenie, ventilyaciya i kondicionirovanie vozduha». (rus.)

2. Russian State Standart GOST R EN 13779-2007. «Ventilyaciya v nezhilyh zdaniyah. Tekhnicheskie trebovaniya $\mathrm{k}$ sistemam ventilyacii i kondicionirovaniya». P. 7 - 8. (rus.)

3. Doklad o sostoyanii okruzhayushchej sredy v gorode Moskve v 2014 godu. Pod red. A.O. Kul'bachevskogo. M.: DPiOOS; NIA-Priroda, 2015. P. 42 - 44. (rus.)

4. Mezhgosudarstvennyj standart GOST 12.1.005-88 «Sistema standartov bezopasnosti truda (SSBT). Obshchie sanitarno-gigienicheskie trebovaniya k vozduhu rabochej zony (s Izmeneniem N 1)». 7 p. (rus.)

5. WHO 2005. Air Quality Guidelines: Global Update 2005. Particulate matter, ozone, nitrogen dioxide and sulfur dioxide. WHO. 2006. P. 484.

6. Vozdejstvie vzveshennyh chastic na zdorov'e. Rekomendacii v otnoshenii politiki dlya stran Vostochnoj Evropy, Kavkaza i Central'noj Azii. Vsemirnaya organizaciya zdravoohraneniya. Evropejskoe regional'noe byuro. 2013. P. 14. (www.euro.who.int). (rus.)

7. Ushakov S.A., Kac YA.G. Ekologicheskoe sostoyanie territorii Rossii. M.: Academia, 2002. 127 p. (rus.)

8. Vladimirov S.N. Transportnye zatory v usloviyah megapolisa. Izvestiya Moskovskogo gosudarstvennogo tekhnicheskogo universiteta MAMI. 2014. 3 (1-19). P. 77 - 84. (rus.)

9. Protasov V.F. Ekologiya, zdorov'e i ohrana okruzhayushchej sredy v Rossii: uchebnoe i spravochnoe posobie. Finansy i statistika, 2009. 521 p. (rus.) 


\title{
Belgorod State Technological University named after V.G.Shukhov, Russia *Corresponding author E-mail: shatalov-victor@bk.ru \\ RELEVANCE OF THE CREATION OF ARTIFICIAL BREATHING ATMOSPHERES IN TERMS OF AIR POLLUTION
}

Shatalov V.V.*, Postgraduate,

\begin{abstract}
: at present, the problem of air quality in large cities with a large number of cars and industrial enterprises is acute. Normative documents on the basis of which the ventilation system is designed regulate the cleaning of the outside air from dust. Purification from gaseous contaminants as well as suspended aerosols such documents do not regulate. According to the results of the study of air quality in Moscow over the past few years, an increased content of pollutants such as $\mathrm{NO}, \mathrm{CO}, \mathrm{CO}_{2}, \mathrm{SO}_{2}$ has been registered. Near busy highways during "rush hours" the vehicles locally "burn" oxygen in the air and emitted products of combustion even more reduce the relative concentration of oxygen in the air. Also, a large number of pollutants come from industrial and agricultural enterprises. The use of additional purification filters in ventilation systems, such as photocatalytic filter, adsorption filter can reduce the concentration of gaseous pollutants to an acceptable minimum, but they will not solve the problem of low oxygen content. Inhalation of air in excess of MPC for a long time may cause irreversible health effects. In this regard, the task of creating artificial respiratory atmospheres becomes relevant. The greatest relevance of air quality and sufficient oxygen content is for children's institutions, as well as special services, the work of which is associated with an increased concentration of attention. The problem of air pollution with aerosols and fine dust has become particularly relevant due to the significant increase in respiratory tract diseases and various allergies.
\end{abstract}

Keywords: air pollution, artificial respiratory atmospheres

Для цитирования: Шаталов В.В. Актуальность создания искуственных дыхательных атмосфер в условиях загрязнения атмосферного воздуха // Строительные материалы и изделия. 2019. Том 2. №3. С. $34-40$. DOI: $10.34031 / 2618-7183-2019-2-3-34-40$

For citation: Shatalov V.V. Relevance of the reation of artificial breathing atmospheres in terms of air pollution. Construction Materials and Products. 2019. 2 (3). P. 34 - 40. DOI: 10.34031/2618-7183-2019-2-3-34-40

Поступила в редакичию 20 февраля 2019 г.

Received: February 15, 2019.

Принята в доработанном виде 1 апреля 2019 г.

Revised: April 1, 2019.

Одобрена для публикаиии 28 апреля 2019 г.

Accepted: April 28, 2019. 